\title{
ON THE BIOMECHANICS OF CYTOKINESIS IN ANIMAL CELLS*
}

\author{
NURI AKKas \\ Department of Mechanical Engineering and Applied Mechanics, University of Michigan, \\ Ann Arbor, Michigan 48109, U.S.A. and Department of Civil Engineering, \\ Middle East Technical University, Ankara, Turkey
}

\begin{abstract}
The material properties of the cell membrane are discussed. Various theories concerning the mechanism of cytokinesis in animal cells are presented. The currently accepted mechanism is that of active muscle-like contraction of the furrow base itself. A mathematical model is developed based on this theory. The cell membrane is modelled as a spherical membrane of nonlinear, elastic material. The membrane undergoes large deformations under the action of a contractile ring force in its equatorial plane. The numerical procedure employed in the solution of the governing equations is explained. The numerical results are compared with the experimental observations available in the literature. It is concluded that the cell membrane stiffness increases during the early stages of cleavage and it, later, decreases. The cell membrane division is a biomechanical instability problem. The factors that may facilitate or block cleavage are discussed. The experimental evidences that support the conjectures of the model are pointed out.
\end{abstract}

\section{INTRODUCTION}

The cell is the basic structural and functional unit of all living organisms. It can simply be defined as a discrete mass of cytoplasm enveloped in a selective and retentive membrane and containing a nucleus. The cytoplasm, a translucent, colloidal material of gelatinous consistency, is composed of numerous differentiated subcellular organelles which carry on the diverse cellular activities, such as growth, division and differentiation. The nucleus, bounded by the nuclear membrane, is the control center of the cell. It contains the chromosomes, the carriers of genetic information. There are virtually thousands of books, monographs and papers written on the biology of cells from which detailed information can be obtained on cytoplasm, organelles, nucleus, and their roles in various stages of cell cycle. For instance, one can be referred to Threadgold (1976), Hall and Baker (1977), Hopkins (1978), Finean et al. (1978), and Avers (1978). On the other hand, for the purposes of our work, it is necessary to discuss the cell membrane and the cell division in more detail.

\section{CELL MEMBRANE}

The cell membrane is the vital biological interface separating the cytoplasm from the external environment. It is a selective, semipermeable barrier through which substances necessary for cellular activities may be acquired and waste products may be removed. The entire complex is a sheet approximately $70 \AA$ in thickness. In the contemporary fluid mosaic model of Singer and Nicolson (1972), the basic structure is a

\footnotetext{
- Received 20 September 1979 ; in revised form 18 January 1980.
}

two-dimensional arrangement of globular integral proteins dispersed in a matrix of fluid lipid bilayer. The membrane is not a tightly stretched layer, but rather loose and wrinkled. It covers fingerlike cytoplasmic processes called microvilli and also lines deep depressions on the cell surface. Water is present in cytoplasm in a concentration between 70 and 85 per cent, Threadgold (1976).

The cell membrane shows the property of a selective permeability. Water-soluble solutes can apparently enter by diffusion through aqueous channels or pores which are 3 to $4 \AA$ in diameter. Indeed, the cell membrane is highly permeable to water. Cells in hypotonic solutions admit water molecules without control and they eventually burst. There is another event in which bursting of the cell membrane can be observed. After a virus enters a cell, a large number of new virus particles is manufactured. Eventually, the cell bursts or splits open which is called death by lysis, Avers (1978). Bursting of the cell membrane means failure of the structure which, in general terms, can be caused by two different but interrelated mechanisms: (a) The membrane material reaches its yield or rupture point, leading to bursting, under the influence of the increasing pressure. (b) The membrane, as a structural system, reaches a point of instability as the intracellular pressure increases. At this point, the cell membrane tends to snap out to another stable equilibrium configuration. However, during this process, the deformations and stresses become so large that the material yields or ruptures, leading to bursting, Akkas (1978). At this time, it is not known which mechanism is the one that initiates the failure of the cell membrane. since the experiments reported in the literature were not undertaken with this purpose in mind.

There are many experiments reported in the literature in which the external force vs some characteristic 
deflection curves of the cell membrane were obtained. They are presented in detail in a review article by Hiramoto (1970). In the suction method, a micropipette connected to a movable reservoir of water is brought up to a cell and when the reservoir is lowered a bulge is sucked out of the cell surface. The pressure vs deformation curves obtained so are approximately linear. Mitchison and Swann (1955) calculated the Young's modulus of the cell membrane of sea urchins using this method. They found a range from $0.91 \times 10^{4}$ to $2.08 \times 10^{4}$ dynes $/ \mathrm{cm}^{2}$. The observed fact that the pressure vs deformation curves are approximately linear does not necessarily imply that the cell membrane material is linear. Because the cell surface is sucked into the micropipette which has a cylindrical inner surface, the final configuration that the bulge can take without being compressed by the cylindrical surface is a hemispherical one. The experiments in which micropipettes are used cannot give that portion of the pressure vs deformation curve which corresponds to unconstrained inflation of the bulge beyond the hemispherical configuration. It has been shown (Akkaş and Engin, 1980) that the nonlinearity of the curve becomes apparent for configurations beyond the hemispherical one.

In the compression method developed by Cole (1932), the cell is compressed between a pair of parallel plates. The force of compression is known and, hence, the intracellular pressure can simply be determined from a consideration of the static equilibrium. The pressure can, then, be related to the tension in the membrane. Cole's results led him to the conclusion that the cell membrane is a thin, elastic membrane. Hiramoto (1963), using this method, estimated the Young's modulus of the cell membrane as $1.2 \times 10^{3}$ dynes $/ \mathrm{cm}^{2}$ for the unfertilized egg. This is one order of magnitude smaller than that obtained by Mitchison and Swann (1955). Yoneda's experimental results (1973), obtained from a modified compression method, indicate that the surface is not even elastic. The surface tension remains constant as the surface area is increased.

As discussed in detail in Hiramoto (1970), various methods of determining rheological properties of the cell yield various force vs deformation curves. In the compression method the force vs deformation curve is concave toward the force axis, whereas in the stretching method it is convex toward the same axis. In the suction method, the curve appears to be linear. These differences are due to the fact that the forces and the deformations measured in the methods do not have a common basis. Plotting the intracellular pressure vs cll volume curve, rather than the external force vs some typical displacement curve, will probably bring the results into, at least qualitative, agreement.

In determining the cell membrane properties, the two most commonly used techniques are the so-called suction and compression methods described above. It is now generally agreed upon, in view of the experimental results obtained from these two methods, that the cytoplasm is enveloped in a thin, elastic membrane. Yoneda (1973) is apparently one of the few who do not accept the concept of an elastic cell membrane. In view of his ex perimental results, Yoneda (1973) supports the liquid-drop concept. As discussed in more detail from a critical viewpoint in Pujara (1978), Yoneda's (1973) conclusion that the surface tension in the cell membrane remains constant irrespective of change in surface area appears to be not valid. Yoneda (1973) initiallly assumes that the surface tension is uniform and remains constant during the compression of the cell. On the other hand, Pujara (1978) showed numerically that the predicted surface tension increases as cell deformation increases. Thus, the findings of the latter author also support the elastic membrane concept.

As mentioned previously, the compression and suction methods yield different elastic moduli for the cell membrane, the difference being one order of magnitude. It is thought to be of interest to discuss the possible reasons causing this apparent discrepancy. It should be realized that the conclusions concerning the mechanical properties of the cell membrane will be affected by the manipulation and interpretation of the experimental data. For instance, as emphasized in Pujara (1978), in suction experiments, whether the cell surface slips, slips partially or does not slip at all over the edge of the pipette will affect the resulting pressure vs deflection curves significantly. In case there is no slip, it is the region of the cell membrane within the pipette only that is stretched. If there is slip, the whole surface of the cell is stretched. In compression experiments, if there is no slip, it is the region of the cell membrane between the two compressing plates that is stretched. Under these circumstances, one should not expect to have good correspondence between the experimental results obtained from the two methods.

An ultrastructural study of the cell membrane may also be helpful in explaining the apparent discrepancies mentioned. It should be noted that the cell 'membrane' that is studied by Mitchison and Swann (1955), Cole (1932), and Hiramoto (1970) is not the bilayer lipid membrane of Singer and Nicolson (1972). The thickness of the lipid bilayer is in the order of several nanometers, whereas the membrane thickness used by the former investigators in their calculations is in the order of a few micrometers. The submembraneous region, corresponding to the 'cortex' of Hiramoto (1970), is considered as part of this membrane. This submembraneous region contains numerous microfilaments which are linked to each other and also to the proteins dispersed in the matrix of the fluid lipid bilayer. The micro-filaments are in a dynamic state; i.e., they are polymerized and depolymerized at various stages of the cell cycle. It is this microfilamentous cortical layer that contributes to the stiffness of the cell membrane. A composite structure may require two or more moduli to characterize it completely and different loading conditions may measure different moduli. In the compression method, the cell membrane is pushed out due to the intracellular 
pressure increase caused by compression. In the suction method, the membrane is sucked out locally. In other words, the pressure difference is applied to most of the cell membrane in the compression test and to only part of the cell membrane by the pipette. Apparently, these two different loading conditions act on the submembraneous region in different manners which may cause some difference in the experimentally observed load-deflection curves.

\section{CYTOKINESIS}

For growth and replacement of cells that wear out, it is essential that new cells are reproduced from old. New cell formation occurs through cell division. It consists of a series of phases in which both nucleus and the extranuclear components of the parent cell undergo a division. Cell division can, in general terms, be said to consist of nuclear division (mitosis) and the division of the cytoplasm and the membrane (cytokinesis). In the present work, mitosis is not considered at all. The literature contains some review articles on cytokinesis. An interested reader is referred to the excellent and recent review articles by Rappaport (1971), which contains 217 references, Arnold (1976), which contains 96 references and concentrates on more recent work, Rappaport $(1974,1975)$, and Schroeder (1975)

Cytokinesis in animal cells appears to be a relatively simple event; namely, division of a membrane, enveloping the cytoplasm, into two. The problem has been to determine the mechanisms of cytokinesis and to distinguish between active and passive phenomena. Accordingly, many theories have been developed to shed some light on these mechanisms. Here, we will present these theories without any detail:

1. Polar expansion: The membrane expands actively at the poles but not the equator. Thus, the equator is passively pushed inward to form the furrow.

2. Polar relaxation: Cleavage is initiated by the relaxation of the membrane in the polar surfaces. Thus, tension in the equatorial surface exceeds that at the poles and the resulting tension difference causes furrowing. It is the polar surfaces where the events causing division take place.

3. Equatorial constriction: The furrow base itself actively contracts. It has independent power to contract. This is a muscle-like contraction of a ring of equatorial surface material.

4. Cytoplasmic streaming: The furrow forms as inner cell contents stream away from the equator and daughter cells are pushed away.

5. Formation of new membrane: The cleavage furrow is formed by the fusion of aligned membraneous vesicles that appear at the equatorial plane

A very detailed discussion of these theories can be found in Rappaport (1971). The currently accepted mechanism of cytokinesis in animal cells is that of active muscle-like contraction of the furrow base itself, and Arnold (1976) dwells solely upon this mechanism.
During division, the mitotic apparatus is believed to play an important role in changing the ultra-structure and mechanical properties of the equatorial surface. Tension in the equatorial surface exceeds that at the poles and the resulting tension difference causes furrowing. It is the equatorial surface where the changes that precipitate cytokinesis occur. During division no new membrane is formed, but the old cell membrane simply extends. The division furrow has a dense ring of circumferentially oriented microfilaments at its leading edge. The microfilaments are fibrils $40-70 \AA$ in dia. and of an indeterminate length. They form a band approx. $0.1-0.2 \mu$ thick and $5-6 \mu$ wide. This band is named the contractile ring. It is the muscle-like contraction of this ring that causes cleavage. It has been proposed that microfilaments associated with surface microvilli constitute the pool from which the constituents of the contractile ring are recruited. The existence of the contractile ring was experimentally confirmed, Schroeder (1975).

\section{MATHEMATICAL MODEL}

The problem of cytokinesis, in contrast to the attention it has received from a biological point of view, has not received much attention from a mechanics point of view. This does not mean that the principles of mechanics have not been made use of in the reports on the subject. Indeed, biologists frequently used basic concepts of mechanics in interpreting their experimental findings. Applications of membrane theories which incorporate neither large deformations nor material nonlinearities (Hiramoto, 1968 and Yoneda, 1973) or calculations based on the assumption that the cell contours during cytokinesis can be fitted to spheres (Ishizaka, 1966 and Yoneda and Dan, 1972) are available. In addition, there are a few other investigations based on a completely fluid model of cleavage dynamics (Greenspan, 1977, 1978), and on the concept of a surface cleavage field ( $\mathrm{Ca}$ talano and Eilbeck, 1978).

Greenspan $(1977,1978)$ treats the cell as a very viscous, homogeneous fluid. He introduces the concept of tension elements which are uniformly distributed over the cell surface before the onset of cleavage. As the uniformity of the surface tension is disturbed. due to some chemical activity within the cell, and the tension elements start moving towards the equatorial surface, cleavage is initiated. The resulting cytoplasmic streaming increases the concentration of the tension elements at the equatorial surface furthering the cleavage. Accordingly, the process is dynamically unstable and once triggered, the cleavage is completed without further stimulus. Greenspan's cleavage mechanism can be sufficient only if the cell membrane has very fiuid characteristics. Cells with stiffer membranes will apparently require an equatorial constriction action. The surface tension elements may stimulate the microfilaments of the cortical layer. It is known that these filaments are all interconnected and are also 
attached to the membrane proteins. It is not clear whether Greenspan's cytoplasmic streaming can cause the breaking (or depolymerization) of these linkages. The breaking of the interconnections among the microfilaments is necessary for an individual filament to flow towards the equator as shown in Fig. 4 of Greenspan (1977). Moreover, it is reasonable to assume that stirring the internal cytoplasm actively during furrowing will offset the cytoplasmic streaming. Thus, according to Greenspan's model, the stirring should affect the furrowing also. However, this is not reported to be the case observed in experiments, Rappaport (1971). The model of Catalano and Eilbeck (1978) is, in principle, different from the mechanical models and, hence, it will not be discussed any further.

To the author's best knowledge, the first and only paper published in literature in which division of the cell membrane has been studied via a mathematical model which incorporates both geometric and material nonlinearities is that by Pujara and Lardner (1979). They examined the deformation pattern of an initially spherical membrane for prescribed displacement at the equator, subject to the condition that the enclosed volume is constant. The nonlinear constitutive relation chosen to describe the behavior of the membrane material is that originally proposed by Skalak et al. (1973). The Mooney-Rivlin material (Green and Adkins, 1970) has also been used, but it is claimed that the former is more satisfactory as far as agreement with experimental results is concerned. The equations used in Pujara and Lardner (1979) are those that govern the large deformations of an initially spherical membrane of nonlinear material and they are presented in detail in Feng and Yang (1973).

Our mathematical model is basically the same as that of Pujara and Lardner (1979). The differences and/or modifications will be clarified during presentation. For the sake of completeness, a sufficiently detailed presentation of the mathematical model and the numerical method used will be given below. A much more detailed analysis can be found in an unpublished thesis, Söylemez (1978). Our mathematical model is developed under the following assumptions:

(a) At the onset of cleavage, the cell membrane has a spherical shape.

(b) In contrast to the assumption used in Pujara and Lardner (1979), the membrane is initially slightly inflated.

(c) Cleavage is caused by contraction of an equatorial ring.

(d) The constitutive relations describing the membrane material are nonlinear, and, moreover, the Mooney-Rivlin material is sufficient.

(e) The viscous properties of the cytoplasm are ignored; hence, the intracellular pressure is uniform.

(f) In contrast to the assumption used in Pujara and Lardner (1979), the volume enclosed by the cell membrane may change. (g) Division of the cell membrane is a large deformation, quasi-static problem.

Assumption (b) is the result of the observations discussed in Hiramoto (1970) where it is stated that there is an initial stretch of the cell surface and it ought to be about $5 \%$. The use of the Mooney-Rivlin material in the model may be the most controversial one. It is generally agreed upon that soft tissues exhibit stress vs stretch ratio diagrams which are exponential in shape. Discussion of various representations can be found, for instance, in Alexander (1968), Hart-Smith and Crisp (1976), and Pujara and Lardner (1978). To try to justify our assumption would be academic, since our numerical results indicate that neither the Mooney-Rivlin representation nor any other exponential representation, in their classical forms, is appropriate for a description of the material properties of the cell membrane during cytokinesis. Later, we will come back to this subject and, indeed, propose a new representation. Exponential forms may be appropriate for red blood cell membranes, but they do not divide.

In their reply to the discussion by Akkas (1980), Pujara and Lardner (1980) present the values of the intracellular pressure variation with cleavage stage. Their results show that the pressure increases monotonically with stage of division. This is in contrast with the results given in Hiramoto (1968). Figure 3 of Hiramoto (1968) clearly shows that ' $\ldots$ at the beginning of cleavage, the pressure increases again and it reaches a peak during cleavage followed by a decrease during the second half of cleavage. The pressure increases by about tenfold during the first half of cleavage and decreases by a similar degree during the second half.' The subject figure is reproduced here as Fig. 1 in a modified form in which only the envelope of Hiramoto's experimental results are given. To see if this apparent discrepancy between Hiramoto's experimental results and the numerical results of Pujara and Lardner (1980) can be resolved by not using the constant volume constraint, we make the assumption $(f)$. Furthermore, this author is of the opinion that it is not absolutely necessary for the cell to have constant

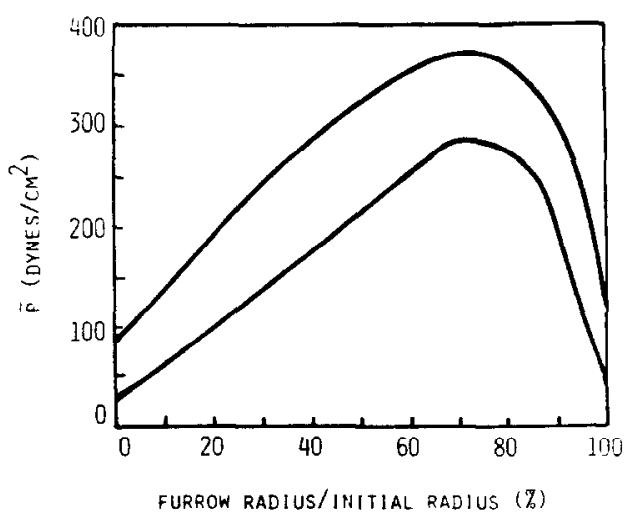

Fig. 1. Envelope of change in intracellular pressure during cleavage. Experimental results of Hiramoto (1968). 
volume during division. The literature on experimental cytology contains works which state that the cell volume roughly remains constant during division. It is well known that the cell surface contains numerous microvilli (ruffles) which are tiny vesicles extending outward and inward from the cell surface. These microvilli, which contain cytoplasmic components, are capable of causing small variations in the cytoplasmic volume. It is also known that they do not remain stationary during division; i.e., at some stages the cell surface has more ruffles and at other stages it is smoother. In addition to the possible contribution of the microvilli to the volume change, a study of the ultrastructural details of the cell membrane reveals that there are other possibilities that may cause slight volume changes. The cell membrane contains numerous channels or pores through which fluid can enter into the cell as well as it can leak out. The possibility of volume increase during cleavage due to inflow of fluid should not be rejected on the ground that the intracellular pressure increase should tend to decrease the cell volume by forcing fluid out. Cell is such a complex system that there still exist many points open to question. For instance, as stated in a previous section, cells in hypotonic solutions admit water molecules without control. During this process, the intracellular pressure increases as well as the cell volume and, eventually, the cells burst open. In other words, a pressure increase in the cell does not necessarily lead to a decrease in cell volume by forcing fluid out. In any case, as pointed out at the beginning of this discussion, it is of practical interest to see if the constant volume constraint during cleavage is the reason behind the apparent discrepancy mentioned.* Under the mentioned assumptions, the problem reduces to the large deformation analysis of a spherical membrane of a nonlinear, elastic material subjected to an equatorial ring load. The governing equations are derived below. Similar derivations can be found in Flügge and Chou (1967), Engin (1976) and Yang and Feng (1970).

Consider an element cut from a membrane of revolution in the deformed configuration. The equilit rium of forces parallel to the axis of the membrane and in the direction of the radius $\bar{\rho}$ of a latitude circle yields the following equations:

$$
\begin{aligned}
\frac{\mathrm{d}}{\mathrm{d} \psi}\left(\bar{T}_{\psi} \bar{\rho} \sin \psi\right)-\bar{p} \bar{\rho} \bar{\rho}_{1} \cos \psi & =0, \\
\frac{\mathrm{d}}{\mathrm{d} \psi}\left(\bar{T}_{\psi} \bar{\rho} \cos \psi\right)-\bar{T}_{\theta} \bar{\rho}_{1}+\bar{p} \bar{\rho} \bar{\rho}_{1} \sin \psi & =0 .
\end{aligned}
$$

In these equations, $\left(\bar{\rho}, \bar{\rho}_{1}, \psi\right)$ define the deformed nonspherical state as shown in Fig. 2. The normal

\footnotetext{
* The numerical results that are given in a later section show that the reason behind this discrepancy is not related to the constant or changing volume condition. One may as well use the constant volume constraint, ignoring the possibly small changes in the total volume during division.
}

pressure acting on the deformed membrane is $\bar{p}$. Finally, $\bar{T}_{\psi}$ and $\bar{T}_{\theta}$ are the membrane forces per unit length of the sides of the deformed element. The deformed configuration $\mathrm{C}$ of Fig. 2 is not known beforehand. Therefore, the quantities occurring in the governing equations are to be expressed in terms of those measured on the uninflated reference configuration A of Fig. 2 which is described by $\left(\bar{r}, \bar{r}_{1}, \phi\right)$. Note that the reference configuration $A$ is not the configuration at which cleavage starts. The configuration at which cleavage starts is B of Fig. 2 which is slightly inflated, but still spherical, in accordance with our assumption (b). The configuration $B$ will be called the initial configuration and the corresponding radius will be called the initial radius. The following relations hold among the quantities of the deformed and reference configurations:

$$
\begin{aligned}
\lambda_{\theta} & =\bar{\rho} / \bar{r}, \quad \lambda_{\phi}=\left(\bar{\rho}_{1} / \bar{r}_{1}\right)(\mathrm{d} \psi / \mathrm{d} \phi), \\
\bar{T}_{\psi} \bar{\rho} & =\bar{N}_{\phi} \bar{r}, \bar{T}_{\theta} \bar{\rho}_{1} \quad \mathrm{~d} \psi=\bar{N}_{\theta} \bar{r}_{1} \mathrm{~d} \phi,
\end{aligned}
$$

in which $\bar{N}_{\phi}$ and $\vec{N}_{\theta}$ are the membrane forces per unit length of the undeformed reference configuration $A$ and $\lambda_{\phi}$ and $\lambda_{\theta}$ are the stretch ratios in the meridional and circumferential directions, respectively. Substituting equations ( 3 ) into equations (1) and (2), we obtain the following equilibrium equations:

$$
\begin{aligned}
\frac{\mathrm{d}}{\mathrm{d} \phi}\left(\bar{N}_{\phi} \bar{r} \sin \psi\right)-\bar{p} r \bar{r}_{1} \lambda_{\phi} \lambda_{\theta} \cos \psi & =0, \\
\frac{\mathrm{d}}{\mathrm{d} \phi}\left(\bar{N}_{\phi} \bar{r} \cos \psi\right)-\bar{N}_{\theta} \bar{r}_{1}+\bar{p} \bar{r} \bar{r}_{1} \lambda_{\phi} \lambda_{\theta} \sin \psi & =0 .
\end{aligned}
$$

The compatibility condition gives an additional relation among the variables:

$$
\frac{\mathrm{d} i_{\theta}}{\mathrm{d} \phi}+\frac{\bar{r}_{1}}{\bar{r}} \lambda_{\theta} \cos \phi-\frac{\bar{r}_{1}}{\bar{r}} i_{\phi} \cos \psi=0 .
$$

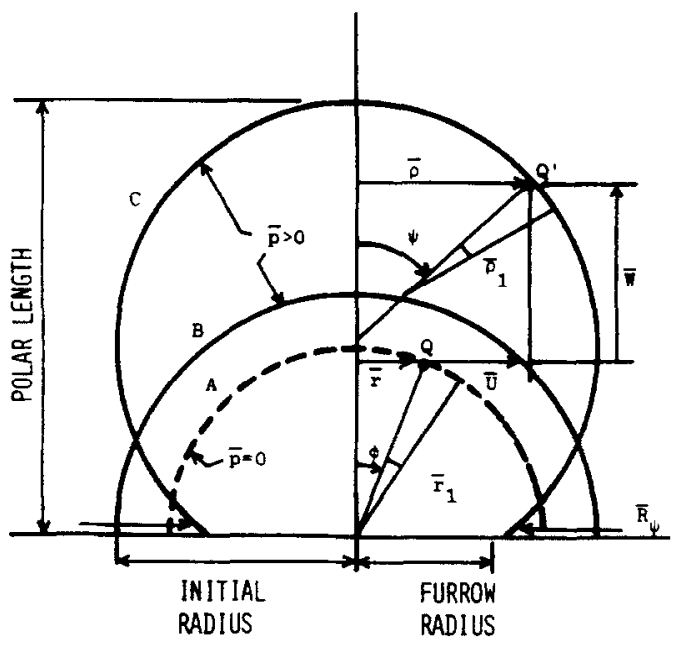

A: ININFLATED REFERENCE CONFIGURATION

B: INFLATED SPHERICAL INITIAL CONFIGURATION

C: DEFORHED MONSPHERICAL CONFIGURATION

Fig. 2. Geometric quantities of deforming cell membrane 
Finally, the constitutive relations for the MooneyRivlin material are

$$
\begin{aligned}
& \bar{N}_{\theta}=\left(2 h C_{1} / \lambda_{\theta}\right)\left(\lambda_{\theta}^{2}-\lambda_{\theta}^{-2} \lambda_{\phi}^{-2}\right)\left(1+\alpha \lambda_{\phi}^{2}\right), \\
& \bar{N}_{\phi}=\left(2 h C_{1} / \lambda_{\phi}\right)\left(\lambda_{\phi}^{2}-\lambda_{\theta}^{-2} \lambda_{\phi}^{-2}\right)\left(1+\alpha \lambda_{\theta}^{2}\right),
\end{aligned}
$$

in which $h$ is the initial uniform thickness of the membrane and $\alpha=C_{2} / C_{1}, C_{1}$ and $C_{2}$ being the material constants. Equations (4-8) are five equations from which the five unknowns $\left(\bar{N}_{\phi}, \bar{N}_{\theta}, \psi, \lambda_{\phi}, \lambda_{\theta}\right)$ of the problem are to be determined. The following quantities will also be needed in the presentation of the results:

The total surface area (of both sides) of the deformed membrane:

$$
\bar{S}=4 \pi \int_{0}^{\pi / 2} \bar{r} \bar{r}_{1} \lambda_{\phi} \lambda_{\theta} \mathrm{d} \phi .
$$

The total enclosed volume (of both sides) of the deformed membrane:

$$
\bar{V}=2 \pi \int_{0}^{\pi / 2} \bar{r}^{2} \bar{r}_{1} \lambda_{\phi} \lambda_{\theta}^{2} \sin \psi \mathrm{d} \phi .
$$

The equatorial ring force per unit length of the deformed membrane:

$$
\bar{R}_{\psi}=2 \bar{N}_{\phi}^{*} \frac{\vec{r}^{*}}{\bar{\rho}^{*}} \cos \left(\pi-\psi^{*}\right), \quad\left(\text { at } \quad \phi=\frac{\pi}{2}\right),
$$

which, when referred to the unit length of the reference membrane, becomes

$$
\bar{R}_{\phi}=2 \bar{N}_{\phi}^{*} \cos \left(\pi-\psi^{*}\right), \quad\left(\text { at } \quad \phi=\frac{\pi}{2}\right) .
$$

The axial force in the equatorial ring:

$$
\bar{F}_{\psi}=\bar{R}_{\psi} \bar{\rho}^{*}=\bar{R}_{\phi} \bar{r}^{*}, \quad\left(\text { at } \quad \phi=\frac{\pi}{2}\right) .
$$

$\bar{R}_{\psi}, \bar{R}_{\phi}$, and $\bar{F}_{\psi}$ are those quantities that occur only in the equatorial plane. The quantities appearing on the right hand sides of equations (11) and (12) are to be evaluated at $\phi=\pi / 2$. That is why they have been superscripted by stars.

Equations (4) through (12) are, now, nondimensionalized as follows:

$$
\begin{array}{rlrl}
r & =\bar{r} / h, \quad r_{1}=\bar{r}_{1} / h, \quad p=\bar{p} / \mathrm{C}_{1}, \\
N_{\phi} & =\bar{N}_{\phi} /\left(\mathrm{C}_{1} h\right), & N_{\theta} & =\bar{N}_{\theta} /\left(\mathrm{C}_{1} h\right), \\
S & =\bar{S} / h^{2}, & V & =\bar{V} / h^{3}, \\
R_{\phi} & =\bar{R}_{\phi} /\left(\mathrm{C}_{1} h\right), & F_{\psi} & =\bar{F}_{\psi} /\left(\mathrm{C}_{1} h^{2}\right) .
\end{array}
$$

The nondimensional equations are not given here separately, because they are similar to the dimensional equations (4)-(12), except that all the barred quantities become unbarred. The resulting equations are valid for any membrane of revolution. If the reference configuration is spherical in shape, which is the case in our work, then $r_{1}=$ constant.

\section{NUMERICAL PROCEDURE}

When the constitutive relations are substituted into the equilibrium equations, the latter, together with the compatibility condition, can be brought to the following form:

$$
\begin{aligned}
& \frac{\mathrm{d} \lambda_{\phi}}{\mathrm{d} \phi}=f_{\phi}\left\langle\phi, \psi, \lambda_{\theta}, \lambda_{\phi}, p\right\rangle, \\
& \frac{\mathrm{d} \lambda_{\theta}}{\mathrm{d} \phi}=f_{\theta}\left\langle\phi, \psi, \lambda_{\theta}, \lambda_{\phi}, p\right\rangle, \\
& \frac{\mathrm{d} \psi}{\mathrm{d} \phi}=f_{\psi}\left\langle\phi, \psi, \lambda_{\theta}, \lambda_{\phi}\right\rangle .
\end{aligned}
$$

Now, one has three equations to determine the three unknowns $\left(\lambda_{\theta}, \lambda_{\phi}, \psi\right)$. The independent variable is $\phi$, and $p$ is the loading parameter. Equations (14) can be solved easily using the Runge-Kutta method (Ralston and Wilf, 1960).

The limits of the in tegrations in equations (14) are $\phi$ $=0$ and $\phi=\pi / 2$. The numerical integration could start at $\phi=\pi / 2$; however, there only $\lambda_{\theta}$ can be specified. Accordingly, the numerical integration must start at $\phi=0$ because, at this point, it is known that $\psi$ $=0$ and $\lambda_{\theta}=\lambda_{\phi}=\lambda_{0} \geq 1.0$. Once a value for $\lambda_{0}$ is selected, the numerical integration can be completed and $\left(\lambda_{\theta}, \lambda_{\phi}, \psi\right)$ can be evaluated at any other location $\phi$, provided that the loading parameter $p$ is specified also. For a selected value of $\lambda_{0}$, there are, indeed, different equilibrium configurations corresponding to various value of $p$

As an example, let us discuss Fig. 3 which gives $p$ vs polar length and $p$ vs furrow radius curves for various values of $\lambda_{0}$. The numerical results presented in Fig. 3 are for $r_{1}=50$ and $\alpha=0.0$. As seen in Fig. 3, for a specified value of the furrow radius, one can find virtually an infinite number of $\left(p, \lambda_{0}\right)$ combinations which satisfy the equilibrium equations. Similarly, for a specified value of the polar length, there are, again, an infinite number of $\left(p, \lambda_{0}\right)$ combinations satisfying the equations. On the other hand, if both the furrow radius and the polar length are specified simultaneously, then there is only one combination of $\left(p, \lambda_{0}\right)$ that satisfies the equilibrium equations. This is where the concept of 'artificial constraint condition' comes into play. The concept is to select those combinations of $\left(p, \lambda_{0}\right)$ which will yield numerical results in agreement with the experimental results as much as possible. For instance, if one can find a relationship between the polar length and the furrow radius during division, that would put a constraint condition on the acceptable combinations of $\left(p, \lambda_{0}\right)$. Such a relationship is available in the literature. Figure 4 gives the envelope of the polar length vs furrow radius curves obtained by Hiramoto (1958) and Yoneda and Dan (1972) during their observations of cleaving sea urchin eggs. Therefore, the multivaluedness of the solution has been eliminated, and unique $\left(p, \lambda_{0}\right)$ combinations can now be obtained. Indeed, these combinations are shown as circles in Fig. 


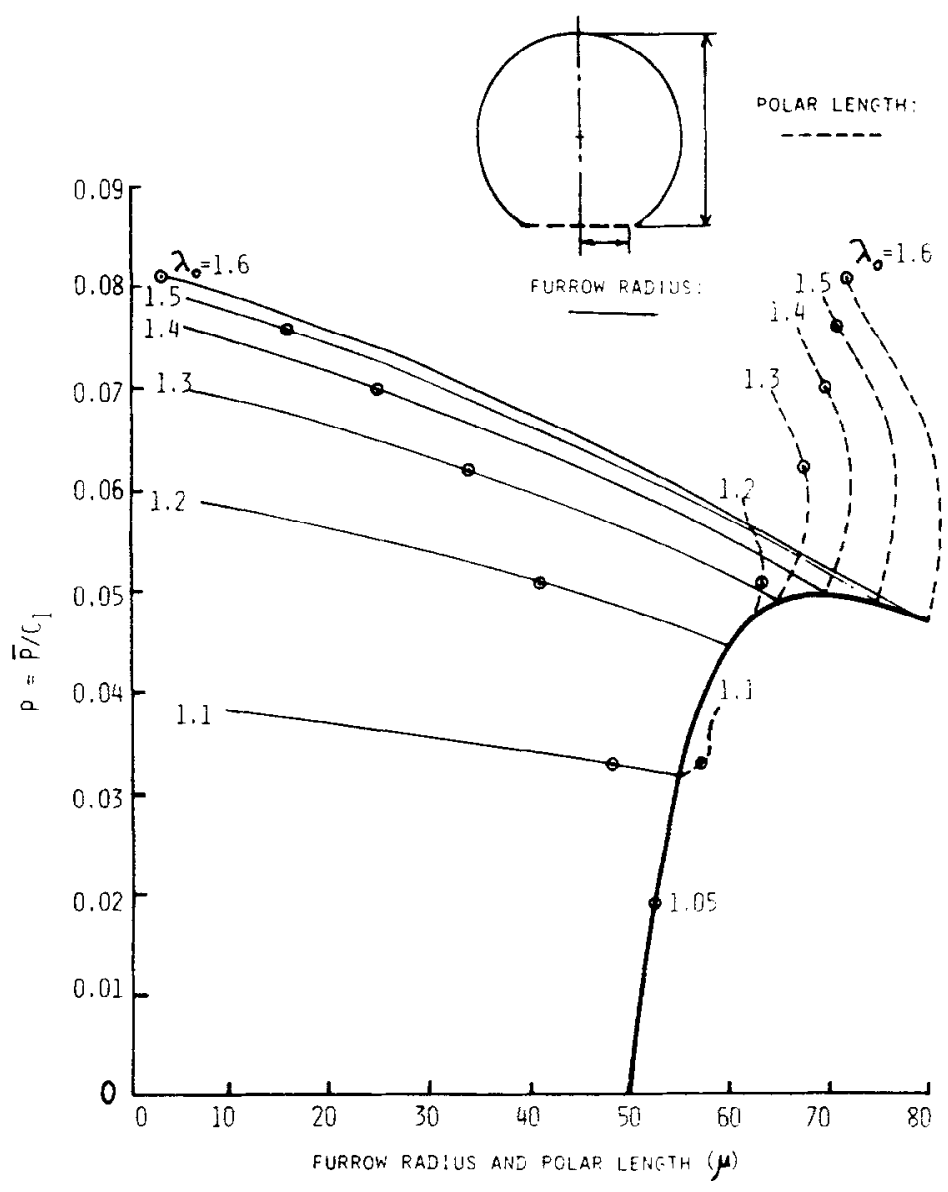

Fig. 3. Intracellular pressure vs furrow radius and polar length for various values of stretch ratio at apex

3 and the resulting polar length vs furrow radius curve is compared with the experimental results in Fig. 4. Pujara and Lardner (1979) obtained similar curves for both the Mooney material and their SZTC material. Their results indicate that the STZC material is a better representation. Our results indicate that the Mooney material representation is also satisfactory. In the numerical solution, the angular increment in the meridional direction was taken to be three degrees. Finally, it is assumed that the membrane cannot be subjected to compressive stresses. Accordingly, when-

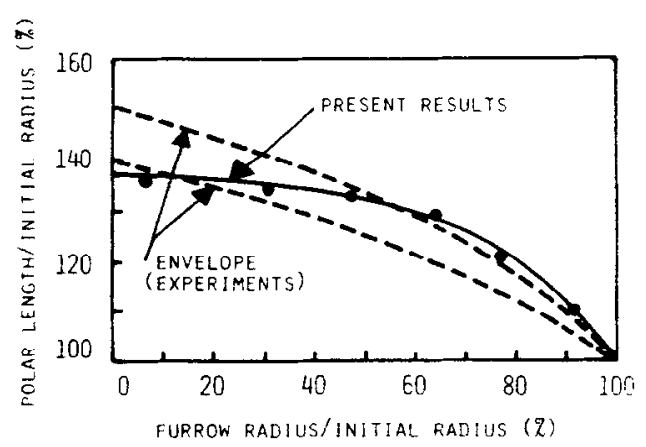

Fig. 4. Change in polar length during cleavage. Envelope of experimental results of Hiramoto (1958) and Yoneda and Dan (1972). ever the stress resultant $N_{\theta}$ turned out to be compressive, which is the case near the equator in the advanced stages of the cleavage, it was set equal to zero. This implies that wrinkling occurs near the equator at these stages.

\section{NUMERICAL RESULTS AND DISCUSSION}

The cell membrane, spherical in shape in its uninflated reference configuration. is assumed to be $100 \mu$ in dia. and $1 \mu$ in effective thickness. Accordingly, the numerical results are for $r_{1}=50$. The second material constant, $C_{2}$, is taken to be zero, which implies that the membrane material is neo-Hookean. In accordance with the observations discussed in Hiramoto (1970), the cell membrane is slightly stretched at the onset of cleavage and the radius of this initial configuration is 1.05 times the radius of the reference configuration.

Figure 5 presents the stretch ratio in the meridional direction, $i_{\phi}$, as a function of the furrow radius. The lower three of the curves are for $\phi=0^{\circ}$ and the upper three are for $\phi=90^{\circ}$. The present results and other available results are all in qualitative agreement. The ratios of the total surface area, $S$, and the total enclosed volume, $V$, of the deformed configuration to the initial surface area, $S_{0}$, and the initial volume, $V_{0}$, are given in Fig. 6. At the end of the cleavage, the total surface area 


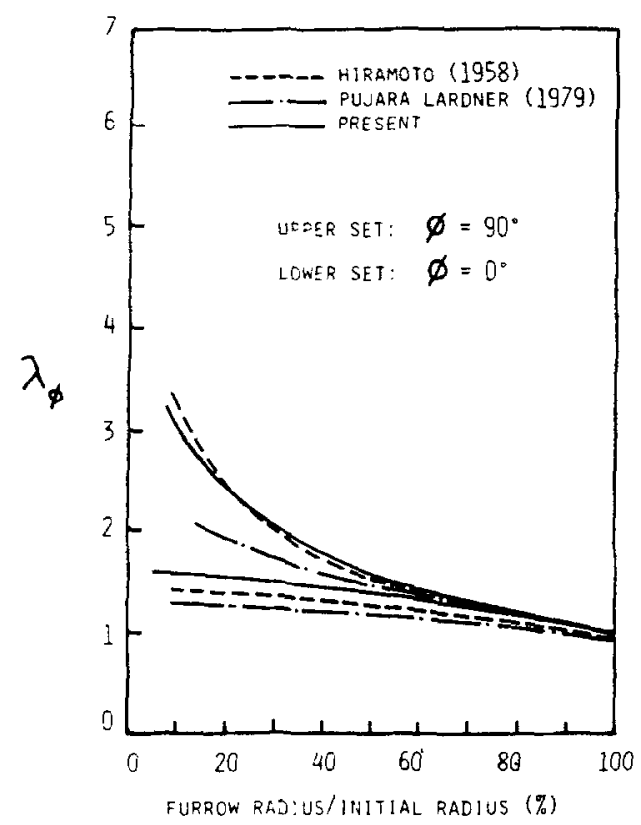

Fig. S. Change in meridional stretch ratio at apex and at equatorial plane during cleavage.

of the two daughter cells is about $25 \%$ larger than that of the parent cell. This is in good agreement with the results obtained by Hiramoto (1958) and Pujara and Lardner (1979). Note also that this number is exactly the same as that mentioned in Rappaport (1971, p. 191). At the initial stages of the cleavage, the total enclosed volume increases slightly, but, as the cleavage is completed, the volume is almost equal to the initial volume. This result is, again, in accordance with the observations concerning the constancy of the cell volume during cytokinesis. In Fig. 7, we have presented the deformed configurations of the cell membrane at three different stages of the division. At the end of the cleavage, the daughter cells are not perfectly spherical in shape.

The intracellular pressure, the membrane force, and the axial force in the equatorial ring are plotted as functions of the furrow radius in Figs. 8, 9, and 10, respectively. The quantities are given in both nondimensional (solid lines) and dimensional (dashed

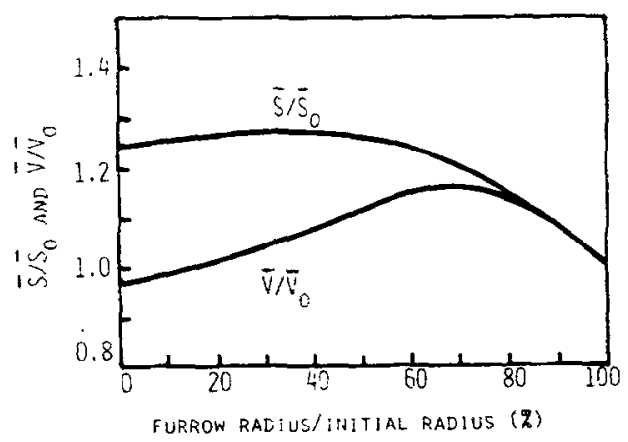

Fig. 6. Change in total surface area and total enclosed volume during cleavage.

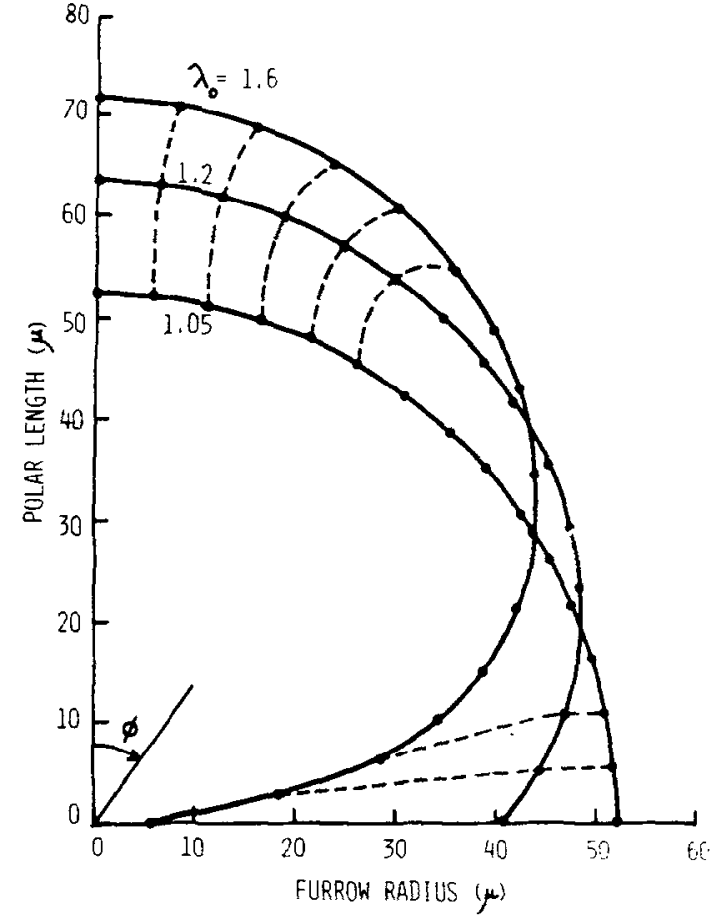

Fig. 7. Deformed configurations of cell membrane at various stages of cleavage.

lines) forms. As seen in Fig. $\delta$, the nondimensional intracellular pressure $p$ increases continuously during cleavage. Recall that the material constant $C_{1}$ is related to the stiffness of the cell membrane. If $C_{1}$ is assumed to remain constant during cleavage, then the pressure $\bar{p}$ in its dimensional form will show the same behavior as the nondimensional pressure $p$; i.e., a continuous increase during cleavage. This result is in total disagreement with the experimental result reported in Hiramoto (1968). As far as the present elastic model is concerned, it is apparent that the only other parameter that can change the shape of the dimensional pressure $\bar{p}$ vs furrow radius curve is the material

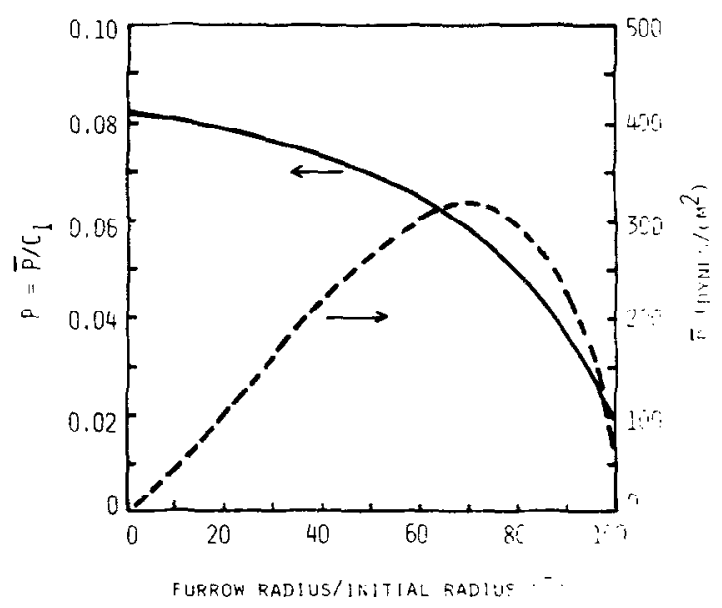

Fig. 8. Change in nondimensional and dimensional in. tracellular pressure during cleavage. 


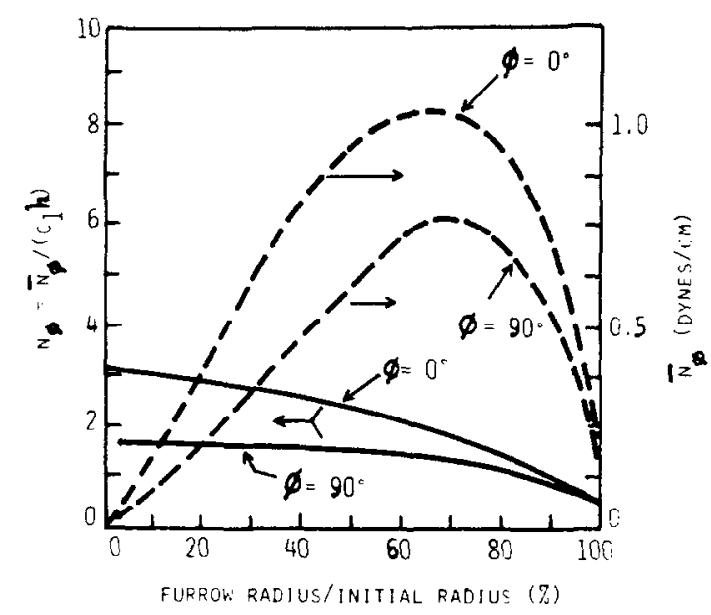

Fig. 9. Change in nondimensional and dimensional membrane force in meridional direction at apex and at equatorial plane during cleavage.

'constant' $C_{1}$. Therefore, for the present numerical results to agree with Hiramoto's experimental results, it is necessary that $C_{1}$ changes during cleavage. The required change in the material 'constant' $C_{1}$ during cleavage is given as a function of both time and furrow radius in Fig. 11. Here, time is that after the onset of cleavage and it is given in Hiramoto (1968). At time is equal to zero, it is assumed that Young's modulus $E$ of the cell membrane material is $1.2 \times 10^{4}$ dynes $/ \mathrm{cm}^{2}$ which is within the range reported by Mitchison and Swann (1955). Since $C_{1}=\mathrm{E} / 6$, this gives $C_{1}=2000$ dynes $/ \mathrm{cm}^{2}$ at the onset of cleavage.

As seen in Fig. 11, the cell membrane becomes stiffer during the early stages of cleavage and, after reaching a limit point, the cell membrane 'deteriorates' as cleavage continues. This is the only way that the numerical predictions of the present elastic model can be brought to a qualitative (and even quantitative) agreement with the available experimental results. This result about the necessity of change of the cell membrane stiffness during cytokinesis was totally unexpected during the

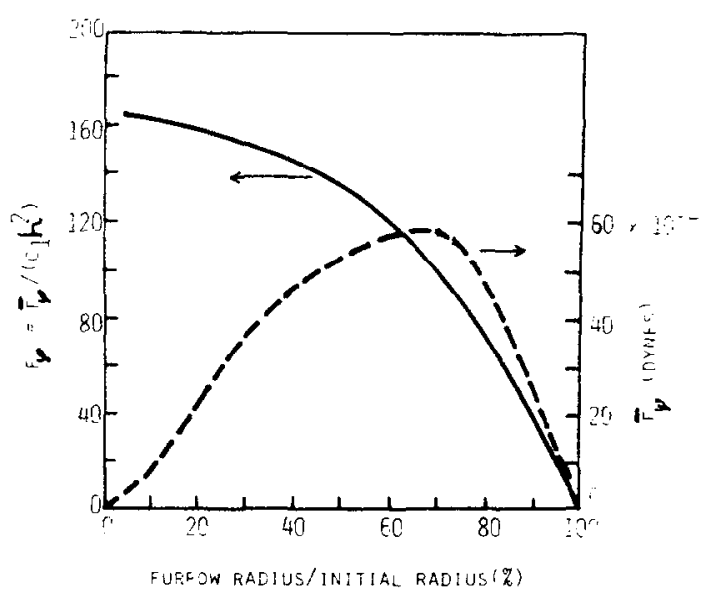

Fig, 10. Change in nondimensional and dimensional total axial ring force during cleavage. initial stages of the mathematical study. Related literature survey revealed that this fact has been. indeed, fairly well established by experiments. In his review article, Hiramoto (1970) clearly states that 'In some eggs, the stiffness reaches a maximum at the beginning of cleavage and falls during and after cleavage .... In some other eggs, the stiffness reaches a minimum at the onset of cleavage, rises during the early half of cleavage and falls during the late half. . A study of geometric variables only, such as $\lambda_{\phi}, S$, or $V$, would not reveal this conclusion. because they are independent of $C_{1}$ explicitly. Pujara and Lardner (1979) did not come to such a conclusion. because their emphasis was mostly on the variation of geometric variables during cytokinesis. Moreover, the only curve that they presented which is directly related to the material 'constant' is incorrect as discussed by Akkaş (1980).

The discussion given above shows that the cell membrane material cannot be modelled as a Mooney material, nor as any other material of similar behavior. in which the material constant $C_{1}$ actually remains constant during division. The material is. first, becoming stiffer and, then, it is deterioraung. The material 'constant' is a function of time. A viscoelastic representation of the material property would probably be more appropriate. However, considering the very slow change of the material property as shown in Fig. 11, we have employed a quasi-static approach in this work. It is assumed that the Mooney material can still represent the cell membrane material, provided that $C$ changes during cytokinesis in accordance with Fig. 11 The dashed, dimensional curves of Figs. 8.9. and 10 have been obtained from the use of the modified Mooney material described above

It should be noted that the device of allowing material constants to vary during clearage may allow a wide variety of materials to give equally good results. The numerical results of Pujara and Lardner (1980) on the intracellular pressure can also be brought into total agreement with Hiramoto's ex perimental results giver. in Fig. 1, employing the described concept of stagedependent elasticity constant. However, the resulting material representation is neither the Mooner material nor the STZC material. What one needs actually is a new material representation which is here called the deteriorating matcrial. The simple device of using a stage-dependent elasticity constant proposed in this work should be considered as a first step towards the development of a better representation. It should also be emphasized that the quasi-static procedure used in this work is not as non-definitive as it may seem. One must select the variation of the elastic material constant with stage of division in such a manner that not only the numerical results on the intracellular pressure and the ring force will agree with the corresponding experimental results, but also the change in membrane stiffness will show the initially increasing and then decreasing behavior observed in the experiments

Before proceeding with the presentation of the 


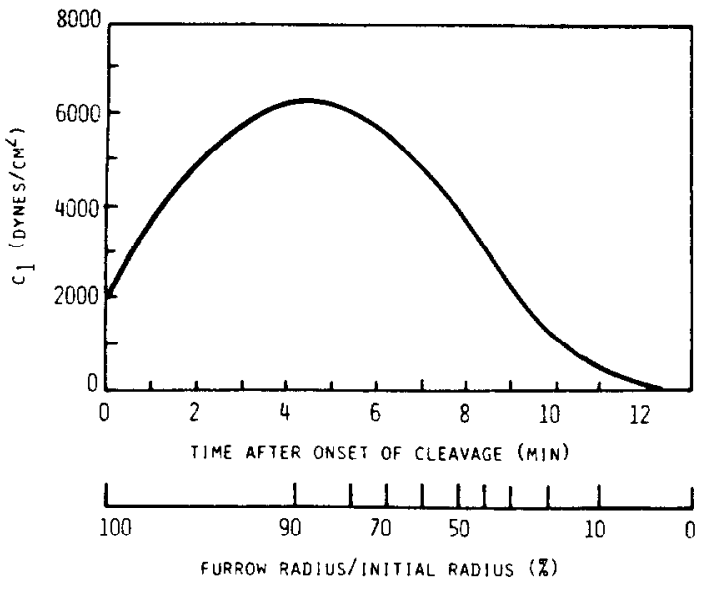

Fig. 11. Predicted change in stiffness of cell membrane during cleavage.

numerical results, it is thought to be of interest to discuss briefly the implications of a viscoelastic model which may prove to be a better representation of the cell membrane during division. Assuming that the cell membrane is a thin, viscoelastic membrane, the development of the governing equations is, in principle, similar to that for the elastic membrane, except that the analysis is complicated by history effects. The previously given equilibrium equations $(4,5)$ and the compatibility condition (6) are still valid. The constitutive relations $(7,8)$ must be replaced by their viscoelastic counterparts. One must be careful in selecting these viscoelastic constitutive relations. The use of linear viscoelastic constitutive equations as the ones described in classical works like Flügge's (1967) may simplify the analysis; however, they are not capable of describing the nonlinearity of the cell membrane material. The concept of material instability is not applicable for such linear material representations. Moreover, the forms of the constitutive equations in Flügge (1967) are not proper for large deformations which are intrinsically the situation in the current work. Nor do they properly account for the requirements of material frame indifference. If the purpose is to see whether the quasi-static approach used in our elastic model described above can be replaced by a viscoelastic approach, one must be consistent in his formulations. In other words, the viscoelastic constitutive equations should be nonlinear as the one studied by Wineman (1978). He models the visco-elastic material by a nonlinear integral constitutive equation which displays Mooney elasticity in its instantaneous and long time equilibrium response limits. The problem will now be governed by a set of nonlinear partial differential-integral equations. The analysis of this viscoelastic formulation should be the topic of further research. When the viscoelastic solution becomes available, it will be possible to discuss the advantages of the viscoelastic model over the quasi-static approach of the present elastic model.

A comparison of the dimensional intracellular pres- sure $\bar{p}$ given in Fig. 8 with the experimental results given in Fig. 1 shows that they are in very good agreement. The dimensional membrane forces, $\vec{N}_{\phi}$, at $\phi=0^{\circ}$ and $\phi=90^{\circ}$ are given as functions of the furrow radius in Fig. 9. A comparison of our Fig. 9 with Hiramoto's (1968) Fig. 8 reveals that the results are in agreement not only qualitatively, but also quantitatively

The dashed curve in Fig. 10 gives the dimensional axial force in the equatorial ring, $\bar{F}_{\psi}$, as a function of the furrow radius. $\bar{F}_{\psi}$ is the only active, external force in our mathematical model. Both the intracellular pressure $\bar{p}$ and the membrane force $\bar{N}_{\phi}$ change passively during cytokinesis, their changes being due to the change in $\bar{F}_{\psi}$. The present result on $\bar{F}_{\psi}$ is in good agreement with the experimental findings of Yoneda and Dan (1972) and Hiramoto (1975). Figure 10 shows clearly that cytokinesis is a biomechanical instability problem; i.e., the dashed curve in the figure has a limit point.

$\tilde{F}_{\psi}$ vs furrow radius curve of Fig. 10 has a limit point at furrow radius-to-initial radius ratio equal to about 0.70 , which we shall call the critical stage of cleavage In actuality, this critical stage cannot easily and clearly be pinpointed, because the curve has a relatively flat portion between the furrow radius-to-initial radius ratio equal to about 0.70 and 0.30 . However, it is certain that the axial force in the equatorial ring, $\bar{F}_{w}$, increases during the early stages of cleavage and decreases during the final stages. Studying Figs. 10 and 11 together, we come to the following inferences about cytokinesis.

Due to some mechanism, the discussion of which is beyond the scope of this work, the axial force in the furrow ring starts increasing. The cell membrane, as if trying to resist this external force (or irritation) which is literally trying to cut the cell into two, gets stiffer. But the ring force increases also. Eventually, the membrane reaches its maximum stiffness capacity and it gives up resisting or it yields. We name the stage after this yielding point as the membrane deterioration stage; the deterioration is not abrupt but rather gradual. After the yielding of the membrane, the ring force does not have to increase any more, rather it decreases gradually. Since the critical stage of cleavage has been reached and passed, division continues and is finally completed.

Now that the concept of a critical stage of cleavage has been noted, one can make some conjectures about cytokinesis based on this concept. If the ring force, for some reason, cannot reach that which corresponds to the critical stage and does not pass it, division will not occur provided that the cell membrane stiffness follows its natural variation with time. If the membrane stiffness does not decrease after reaching the critical stage, for division to occur it is necessary that the ring force increase continuously. In this case, the microfibrils may break in which case the cell will return to its initial shape since, now, there is no ring force squeezing it. If the membrane stiffness does not show its initial 
increase during the very early stages of division but, rather, decreases continuously, then very small ring force is sufficient to complete the division. This may be termed as an uncontrolled division. If the furrow ring is destroyed by some external means before the critical stage of cleavage is passed, the division will stop and the membrane will turn back to its initial configuration. If the destruction of the ring is affected after the critical stage has been passed, the division will continue. Here, it is assumed that the external means used for the destruction of the contractile furrow ring does not affect the natural behavior of the cell membrane.

The following observations are from Rappaport (1971):

A chemical agent, Cytochalasin B, rapidly destroys an equatorial array of circumferentially arranged fibrils and it stops furrowing almost immediately. When the furrow of a partly divided egg is cut from the inside or torn, the cell quickly resumes its initial spherical form.

The following observations are from Arnold (1976). The inserts in parentheses are ours:

The contractile ring in cleaving eggs is a transitory structure, existing for only six and seven minutes at $20^{\circ} \mathrm{C}$. As contraction proceeds, the contractile ring decreases in volume. As cleavage continues (very likely after the critical stage has been passed), actincontaining filaments from the contractile ring may well disassemble and they are recruited for utilization elsewhere in the cell. (This may be considered to correspond to the gradual decrease in the total axial ring force during the second half of cleavage.)

These observations support some of our conjectures. At this time, the author is unable to present any experimental evidence that would support the remaining conjectures. For instance, will the, say, 80 or $90 \%$ cleaved cell return to its initial configuration when its furrow ring is destroyed? Do the chemical agents that block cleavage without destroying the furrow ring increase the stiffness of the cell membrane? Do the agents that facilitate furrowing, such as calcium, decrease the cell membrane stiffness? There are still many questions to be answered. It is hoped that future investigations on the biomechanics of cytokinesis will shed more light on this exciting field.

\section{SL:MMARY}

After a brief presentation of the material properties of the cell membrane and of the hypotheses concerning cytokinesis in animal cells, a mathematical model describing this phenomenon was developed. The model is a spherical membrane of an elastic, nonlinear material and undergoing large deformations due to a contractile, equatorial ring force. The numerical procedure employed was explained. The numerical results showing the variations of the quantities during cytokinesis were given and discussed. According to the results, the cell membrane stiffness increases during the early stages of cytokinesis and it decreases later Cytokinesis can be considered as a biomechanical instability problem. Based on the conclusions of the numerical results, some conjectures concerning cytokinesis were made. Experimental evidences available in the literature that support some of the conjectures were pointed out. All the numerical results obtained are in qualitative, and most of the time, quantitative, agreement with experimental results.

Acknowledgement - Some parts of this investigation were carried out while the author was a Visiting Fulbright Scholar at the University of California, Berkeley, California, USA.

\section{REFERENCES}

Akkas, N. (1978) On the dynamic snap-out instability of infiated nonlinear spherical membranes. Int.J. nonl. Mech $13,177-183$

Akkas, N. (1980) Letter to the editor. J. Biomechanics. 13, 459-466. Vol. 13, 459-460.

Akkas, N. and Engin, A. E. (1980) On the etiology and biomechanics of hernial sac formation. To be published.

Alexander, H. (1968) A constitutive relation for rubberlike materials. Int. J. engng Sci. 6, 549-563.

Arnold, J. M (1976) Cytokinesis in animal cells: new answers to old questions. In The Cell Surface in Animal Em bryogenesis and Development (Edited by G. Poste and G. L. Nicolson), Elsevier-North Holland Biomedical Press, 55-80.

Avers, C. J. (1978) Busic Cell Bloloys: D Van Nostrand. Nex York

Catalano, G. and Eilbeck, J. C. (1978) A mathematıcal model for embryonic cell division based on a surface "cleavage field". J. theor. Biol. 75, 123-137.

Cole, K. S. (1932) Surface forces of the Arbacia egg. J. Cell Comp. Phisiol 1, 1-9.

Engin, A. E. (1976) On the large deformation theory of flurdfilled shells of revolution. Shock and l'ith Dig. 8, No. 8 , $35-47$.

Feng, W.W. and Yang. W. H. (1973) On the contact problem of an inflated spherical nonlinear membrane. $J$ appl Mech $40,202-214$

Finean, J. B., Coleman, R. and Michell, R. H (1978) Membranes and their Cellular Functions ind edn. Blackwell, Oxford

Flügge, W. (1967) Viscoelasticiti. Blaisdell Publ. Co., Wal tham, Massachusetts

Flügge, W and Chou, S. C. (1967) Large-deformation theory of shells of revolution. J. appl. Mech 34, 56-58

Green, A. E and Adkins, J. E 11970$)$ Large Elustic Deformations, 2nd edn. Oxford Liniversity Press. London.

Greenspan, H. P. (1977) On the dvnamics of cell cleavage. $J$ theor. Biol 65, 79-99.

Greenspan, H. P. (1978) On fiud-mechanical simulations of cell division and movement. J. theor. Biol. 70, 125134

Hall, J. L. and Baker, D. A (1977) Cell Membrames and lon Transport. Longman, London

Hart-Smith, L. J. and Crisp, J. D. C. (1976) Large elastic deformations of thin rubber membranes. $I m$ J. enyng. So $5,1-24$.

Hiramoto, Y (1958) A quantitative description of protoplasmic movement during cleavage in the sea urchin egg. Exp. Biol. 35, 407-424

Hiramoto, Y. (1963) Mechanical properties of sea urchin eggs. II. Changes in mechanical properties from fertilization to cleavage. Expl. cell. Res. 32, 76-89.

Hiramoto. Y 11968$)$ The mechanics and mechanism of cleavage in the sea urchin egg. Simp Su Exp Bin 22. $311 \div 327$ 
Hiramoto, Y. (1970) Rheological properties of sea urchin eggs. Biorheology 6, 201-234.

Hiramoto, Y. (1975) Force exerted by the cleavage furrow of sea urchin eggs. Dev. Growth Diff. 17, 27-38.

Hopkins, C. R. (1978) Structure and Function of Cells. W. B. Saunders, London.

Ishizaka, S. (1966) Surface characteris of dividing cells. II. Isotropy and uniformity of surface membrane. J. exp. Biol. 44, 225-232.

Mitchison, J. M. and Swann, M. M. (1955) The mechanical properties of the cell surface. III. The sea urchin egg from fertilization to cleavage. J. exp. Biol. 32, 734-750.

Pujara, P. (1978) Analysis of Finite Deformations of Membranes. Unpublished $\mathrm{Ph}$.D Thesis, University of Illinois at Urbana-Champaign, Urbana, Illinois (Directed by T. J. Lardner).

Pujara, P. and Lardner, T. J. (1978) Deformations of elastic membranes - effect of different constitutive relations. $Z$. angew. Math. Phy's. 29, 315-327.

Pujara, P. and Lardner, T. J. (1979) A model for cell division. J. Biomechanics 12, 293-299. Also see Akkas, N. (1980).

Pujara, P. and Lardner, T. J. (1980) Reply. J. Biomechanics. $13,460-461$

Ralston, A. and Wilf, H. (1960) Mathematical Methods for Digital Computers. pp. 110-120. Wiley, New York.

Rappaport, R. (1971) Cytokinesis in animal cells. Int. Ret. Cytol. 31, 169-213.

Rappaport, R. (1974) Cleavage. In Concepts of Development (Edited by J. Lash and J. R. Whittaker) pp. 76-98. Sinauer, Connecticut.
Rappaport, R. (1975) Establishment and organization of the cleavage mechanism. In Molecules and Cell Motement (Edited by S. Inoué and R. E. Stephens) pp. 287-304. Raven Press, New York.

Schroeder, T. E. (1975) Dynamics of the contractile ring. In Molecules and Cell Movement (Edited by S. Inoué and R. E. Stephens) pp. 305-334. Raven Press, New York.

Singer, S. J. and Nicolson, G. L. (1972) The fluid mosaic model of the structure of cell membranes. Science 175, 720.

Skalak, R., Tözeren, A., Zarda, R. and Chien, S. (1973) Strain energy function of red blood cell membranes. Biophys. $J$. 13, 245-264.

Söylemez, H. (1978) Division of a Nonlinear Spherical Membrane. Unpublished MS Thesis, Department of Civil Engineering, Middle East Technical University, Ankara, Turkey (Directed by N. Akkaş).

Threadgold, L. T. (1976) The Ultrastructure of the Animal Cell. 2nd edn. Pergamon Press, Oxford.

Wineman, A. (1978) Bifurcation of response of a nonlinear viscoelastic spherical membrane. Int. J. Solids Structures. 14, 197-212.

Yang, W. H. and Feng, W. W. (1970) On axisymmetrical deformations of non-linear membranes. J. appl. Mech. 37, 1002-1011.

Yoneda, M. (1973) Tension at the surface of sea urchin eggs on the basis of 'liquid-drop' concept. Adt. Biophys. 4, 153-190.

Yoneda, M. and Dan, K. (1972) Tension at the surface of the dividing sea urchin egg. J. exp. Biol. 57, 575-587. 